\title{
A Utility-Based Congestion Control Scheme for Internet-Style Networks with Delay
}

\author{
Tansu Alpcan ${ }^{1}$ and Tamer Başar ${ }^{1}$ \\ (alpcan, tbasar)@control.csl.uiuc.edu
}

\begin{abstract}
In this paper, we develop, analyze and implement a congestion control scheme obtained in a noncooperative game framework where each user's cost function is composed of a pricing function, proportional to the queueing delay experienced by the user, and a fairly general utility function which captures the user demand for bandwidth. Using a network model based on fluid approximations and through a realistic modeling of queues, we establish the existence of a unique equilibrium as well as its global asymptotic stability for a general network topology. We also provide sufficient conditions for system stability when there is a bottleneck link shared by multiple users experiencing non-negligible communication delays. Based on these theoretical foundations, we implement a window-based, end-to-end congestion control scheme, and simulate it in $n s-2$ network simulator on various network topologies with sizable propagation delays.

Methods Keywords: Control theory, Mathematical programming/optimization, Simulations, Economics.
\end{abstract}

\section{INTRODUCTION}

Game theory provides a natural framework for developing pricing and congestion control mechanisms for the Internet. Users on the network can be modeled as players in a congestion control game where they choose their strategies or in this case flow rates. Players are noncooperative in terms of their demands for network resources, and have no specific information on other users' strategies. A user's demand or utility for bandwidth is captured in a utility function, and may not be bounded. To compensate for this, one can devise a pricing function, proportional to the bandwidth usage of a user, in order to preserve the network resources and to provide an incentive for the user to implement end-to-end congestion control [1]. A useful concept in such a noncooperative congestion control game is that of Nash equilibrium [2] where each player minimizes his/her own cost (or maximize payoff) given all other players' strategies. There is rich literature on game theoretic analysis of flow control problems utilizing both cooperative [3] and noncooperative [4], [5], [6] frameworks. Congestion control schemes utilizing pricing schemes based on explicit feedback have been proposed by Kelly et al. [7], [8], and Gibbens et al. [9], and subsequent studies have further elaborated on this approach following its basic principles [10], [11], [12].

\footnotetext{
${ }^{0}$ Research supported in part by the NSF grant CCR 00-85917. Al correspondence should be forwarded to: Prof. Tamer Başar, Coordinated Science Laboratory, University of Illinois, 1308 West Main Street, Urbana, IL 61801-2307 USA. Tel: (217) 333-3607; Fax: (217) 244-1653; E-mail: tbasar@control.csl.uiuc.edu

${ }^{1}$ Coordinated Science Laboratory and Department of Electrical and Computer Engineering, University of Illinois, 1308 West Main Street, Urbana, IL 61801 USA.
}

Although the game theoretic approach provides a suitable framework for formulating and studying congestion and flow control problems in general networks, there are some inherent restrictions on implementable cost functions in the case of Internet-style networks. For example, the current structure of the Internet makes it difficult, if not impossible, for users to obtain detailed real time information on the state of the network and on other users. Therefore, users are bound to use indirect aggregate metrics that are available to them, such as packet drop rate and variations in the average round trip time (RTT) of packets in order to infer the current situation in the network. Packet drops, for example, are currently used by most widely deployed versions of TCP as an indication of congestion. In this paper, however, we propose and analyze a pricing and congestion control scheme based on variations in the queueing delay a user experiences. A similar approach has been suggested in a version of the transfer control protocol (TCP), known as TCP Vegas [13]. Although TCP Vegas is more efficient than a widely used version of TCP, TCP Reno [14], the suggested improvements are empirical and based on experimental studies. Another study, by Mo and Walrand [12], also makes use of an approach similar to the one in this paper; however, it is based on fairness and pricing concepts of Kelly, and employs only a narrow set of utility functions in describing user demands.

The noncooperative congestion control game introduced in this paper is characterized by a cost function for each user that is defined as the difference of pricing and utility functions. The pricing function is proportional to the queueing delay experienced by the user, whereas the utility function that quantifies the user demand for bandwidth belongs to a broad class of strictly increasing and strictly concave functions. Through a network model based on fluid approximations, and a realistic queueing model, we show the existence of a unique 'Nash' equilibrium, under the assumption that the effect of a user's flow on congestion cost is vanishingly small, which holds especially if the number of users is large. Furthermore, we establish the global stability of the equilibrium under a general network topology. We also investigate stability of the system in a network with non-negligible propagation delays, and provide sufficient conditions for stability in the case of a bottleneck node with multiple users. Based on the theoretical foundations developed, we design a window-based, end-to-end congestion control scheme for Internet-style networks, which is TCP-friendly [15]. This congestion control scheme is then simulated in Network Simulator $2(n s-2)$ over Internet protocol 
(IP) for various network topologies.

The rest of the paper is organized as follows: The underlying network model and cost function are given in the next section. In Section III, the existence of a unique equilibrium and global stability of the system under a general network topology are established. Section IV generalizes the stability analysis of Section III to the case with delay, with a single bottleneck link. In Section $\mathrm{V}$ we provide a realistic implementation of the congestion control scheme for IP networks. Section VI includes simulation results, and is followed by the concluding remarks of Section VII.

\section{THE MODEL}

\section{A. The Network Model}

We consider a general network model based on fluid approximations. Fluid models are widely used in addressing a variety of network control problems such as congestion control [12], [5], [16], routing [5], [6], and pricing [7], [3], [17]. The topology of the network is characterized by a set of nodes $\mathcal{N}=$ $\{1, \ldots, N\}$ and a set of links $\mathcal{L}=\{1, \ldots, L\}$, connecting the nodes. In this network model, we make the natural assumption of connectivity, and let $\mathcal{M}:=\{1, \ldots, M\}$ denote the set of active users. Each link $l \in \mathcal{L}$ has a fixed capacity $C_{l}>0$, and an associated buffer size $b_{l} \geq 0$. For simplicity, each user is associated with a (unique) connection. Hence, the $i^{\text {th }}$ $(i \in \mathcal{M})$ user corresponds to a unique connection between the source and destination nodes, $s_{i}, d e_{i} \in \mathcal{N}$, and we denote the corresponding route (path), which is a subset of $\mathcal{L}$, by $R_{i}$. The nonnegative flow, $x_{i}$, sent by the $i^{\text {th }}$ user over this path $R_{i}$ satisfies the bounds $0 \leq x_{i} \leq x_{i, \max }$. The upper bound, $x_{i, \max }$, on the $i^{t h}$ user's flow rate may be a user specific physical limitation, and cannot exceed the minimum capacity of the links on the route, $\min _{l}\left\{C_{l}, l \in R_{i}\right\}$.

It is possible to define a routing matrix, $\mathbf{A}$, as in [7] that describes the relation between the set of routes $\mathcal{R}$ associated with the users (connections) and links $l \in \mathcal{L}$ :

$$
A_{l, i}=\left\{\begin{array}{lll}
1, & \text { if source i uses link } l & i \in \mathcal{M} \text { and } \\
0, & \text { if source i does not use } \operatorname{link} l & l \in \mathcal{L}
\end{array}\right.
$$

Using the routing matrix $\mathbf{A}$, the capacity constraints of the links are given by

$$
\mathbf{A x} \leq \mathbf{C}
$$

where $\mathbf{x}$ is the $(M \times 1)$ flow rate vector of the users and $\mathbf{C}$ is the $(L \times 1)$ link capacity vector. If the aggregate sending rate of users whose flows pass through link $l$ exceeds the capacity, $C_{l}$, of the link then the arriving packets are queued (generally on a first-come first-serve basis) in the buffer, $b_{l}$, of the link with $b_{l, \max }$ being the maximum buffer size. Let the total flow on link $l$ be given by $\bar{x}_{l}:=\sum_{i: l \in R_{i}} x_{i}$. Thus, the buffer level at link $l$ evolves in accordance with

$$
\dot{b}_{l}(t)= \begin{cases}{\left[\bar{x}_{l}-C_{l}\right]^{-},} & \text {if } b_{l}(t)=b_{l, \max } \\ \bar{x}_{l}-C_{l}, & \text { if } 0<b_{l}(t)<b_{l, \max } \\ {\left[\bar{x}_{l}-C_{l}\right]^{+},} & \text {if } b_{l}(t)=0\end{cases}
$$

where $\dot{b}_{l}(t)$ denotes $\left(\partial b_{l}(t) / \partial t\right),[.]^{+}$represents the function $\max (., 0)$, and [.]- represents the function $\min (., 0)$.

\section{B. The Cost (Objective) Function}

An important indication of congestion for internet-style networks is the variation in queueing delay, $d$, which is defined as the difference between the actual delay experienced by a packet, $d^{a}$, and the fixed propagation delay of the connection, $d^{p}$. If the incoming flow rate to a router exceeds its capacity, packets are queued (generally on a first-come first-serve basis) in the existing buffer of the router, leading to an increase in the RTT of packets. Hence, RTT on a congested path is longer than the base RTT, which is defined as the sum of propagation and processing delays on the path of a packet. The queueing delay at the $l^{t h}$ link, $d_{l}$, is a nonlinear function of the excess flow on that link, and is given by

$$
\dot{d}_{l}(\mathbf{x}, t)= \begin{cases}{\left[\frac{1}{C_{l}}\left(\bar{x}_{l}-C_{l}\right)\right]^{-},} & \text {if } d_{l}(t)=d_{l, \max } \\ \frac{1}{C_{l}}\left(\bar{x}_{l}-C_{l}\right), & \text { if } 0<d_{l}(t)<d_{l, \max } \\ {\left[\frac{1}{C_{l}}\left(\bar{x}_{l}-C_{l}\right)\right]^{+},} & \text {if } d_{l}(t)=0\end{cases}
$$

in accordance with the buffer model described in (3), with $d_{l, \max }$ being the maximum possible queueing delay. Thus, the total queueing delay, $D_{i}$, a user experiences is the sum of queueing delays on its path, namely $D_{i}(\mathbf{x}, t)=$ $\sum_{l \in R_{i}} d_{l}(\mathbf{x}, t), i \in \mathcal{M}$.

Let us define a cost function for each user as the difference between pricing and utility functions. The pricing function of the $i^{t h}$ user is linear in $x_{i}$, and is proportional to the total queueing delay $D_{i}(t)$ of the user. The utility function $U_{i}\left(x_{i}\right)$ is assumed to be strictly increasing, differentiable, and strictly concave; it basically describes the user's demand for bandwidth. Accordingly, we make use of variations in RTT to devise a congestion control and pricing scheme. The cost (objective) function for the $i^{t h}$ user at time $t$ is thus given by

$$
J_{i}(\mathbf{x}, t)=\alpha_{i} D_{i}(\mathbf{x}, t) x_{i}-U_{i}\left(x_{i}\right)
$$

which s/he wishes to minimize. In accordance with this objective, we consider a simple dynamic model of the network game where each user changes her/his flow rate in proportion with the gradient of her/his cost function with respect to her/his flow rate, $\dot{x}_{i}=-\partial J_{i}(\mathbf{x}) / \partial x_{i}$. Thus, the update algorithm for the $i^{\text {th }}$ user is:

$$
\dot{x}_{i}= \begin{cases}{\left[\frac{d U_{i}\left(x_{i}\right)}{d x_{i}}-\alpha_{i} D_{i}(\mathbf{x}, t)\right]^{-},} & \text {if } x_{i}=x_{i, \max } \\ \frac{d U_{i}\left(x_{i}\right)}{d x_{i}}-\alpha_{i} D_{i}(\mathbf{x}, t), & \text { if } 0<x_{i}<x_{i, \max } \\ {\left[\frac{d U_{i}\left(x_{i}\right)}{d x_{i}}-\alpha_{i} D_{i}(\mathbf{x}, t)\right]^{+},} & \text {if } x_{i}=0\end{cases}
$$

where the effect of the $i^{t h}$ user on the delay, $D_{i}(\mathbf{x}, t)$, s/he experiences is ignored. This assumption can be justified for networks with a large number of users, where the effect of each user is vanishingly small. Furthermore, from a practical point of view, it is extremely difficult if not impossible for a user to estimate her/his own effect on queueing delay. 


\section{Stability AnAlysis}

In this section, we analyze the stability of the system described by (4) and (6). First, we investigate the simple case of a single link with a single user in order to gain further insight into the system. ${ }^{1}$ We then generalize the analysis to a single link with multiple users. Finally, we establish stability for a general network topology with multiple links and users.

\section{A. Stability for a Single Link with a Single User}

For a single user on a single link, the equations describing the dynamics of the system consist of the user algorithm, which is a simplified version of (6), and queueing delay equation for a single user derived from (4). For the time being we ignore the effects of boundaries on the system:

$$
\begin{aligned}
& \dot{x}(t)=\frac{d U(x)}{d x}-\alpha d(x, t) \\
& \dot{d}(t)=\frac{x}{C}-1
\end{aligned}
$$

where $d$ is the queueing delay, $x$ is the user flow rate, and $C$ is the link capacity.

The system (7) has a unique equilibrium point $\left(x^{*}, d^{*}\right)$ given by $x^{*}=C$ and $d^{*}=(1 / \alpha) d U\left(x^{*}\right) / d x$. We implicitly assume that $\alpha$ is chosen sufficiently high such that the equilibrium point is feasible, $d^{*}<d_{\max }$. Defining the queueing delay and flow rate around the equilibrium point, $\tilde{x}:=x-x^{*}$ and $\tilde{d}:=d-d^{*}$, we obtain the following equivalent system around the equilibrium:

$$
\begin{aligned}
\dot{\tilde{x}}(t) & =g(\tilde{x})-\alpha \tilde{d}(t), \\
\dot{\tilde{d}}(t) & =\frac{1}{C} \tilde{x},
\end{aligned}
$$

where the function $g(\tilde{x})$ is defined as

$$
g(\tilde{x}):=\frac{d U(x)}{d x}-\frac{d U\left(x^{*}\right)}{d x} .
$$

Note that

$$
g(\tilde{x}) \begin{cases}>0 & , \text { if } \tilde{x}<0 \\ <0 & , \text { if } \tilde{x}>0 \\ =0 & , \text { if } \tilde{x}=0\end{cases}
$$

due to the fact that $U(x)$ is strictly concave in $x$, and hence, $(d U(x) / d x)$ is strictly decreasing.

The system (8) can be viewed as a generalized pendulum equation with $g(\tilde{x})$ as the friction term [18]. Let us define a set $\tilde{\Omega}$ as

$$
\begin{array}{r}
\tilde{\Omega}=\left\{(\tilde{x}, \tilde{d}) \in \mathbb{R}^{2}:-x^{*} \leq \tilde{x} \leq x_{\max }-x^{*}\right. \\
\text { and } \left.-d^{*} \leq \tilde{d} \leq d_{\max }-d^{*}\right\},
\end{array}
$$

where $d_{\max }$ and $x_{\max }$ are finite upper-bounds on $d$ and $x$ respectively.

Next define an energy-like Lyapunov function on the set $\tilde{\Omega}$

$$
V(\tilde{x}, \tilde{d})=\frac{1}{\alpha}(\tilde{x})^{2}+C(\tilde{d})^{2} .
$$

\footnotetext{
${ }^{1}$ Admittedly, in this case the assumption of an individual user not affecting the delay on a link is violated, but still this exercise is useful for the subsequent analysis dealing with the multiple users case.
}

Notice that $V(\tilde{x}, \tilde{d})$ is positive definite on $\tilde{\Omega}$. The derivative of $V$ along the system trajectories is given by

$$
\dot{V}(\tilde{x}, \tilde{d})=\frac{2}{\alpha} g(\tilde{x}) \tilde{x} \leq 0
$$

where the inequality follows from (9). Thus, $\dot{V}(\tilde{x}, \tilde{d})$ is negative semi-definite. Let $S:=\{(\tilde{x}, \tilde{d}) \in \tilde{\Omega}: \dot{V}(\tilde{x}, \tilde{d})=0\}$. It follows from (9) that $S=\{(\tilde{x}, \tilde{d}) \in \tilde{\Omega}: \tilde{x}=0\}$. Hence, for any trajectory of the system that belongs to $S$, we have $\tilde{x} \equiv 0$. It follows then directly from (8) that

$$
\tilde{x} \equiv 0 \Rightarrow \dot{\tilde{x}}=0 \Rightarrow g(\tilde{x})=0 \Rightarrow \tilde{d}=0 .
$$

Therefore, the only solution that can stay identically in $S$ is the zero solution, which corresponds to the unique equilibrium of the original system (7). We next consider the effect of boundaries as described by (4) and (6) with $\tilde{d}=d_{l}$ and $\tilde{x}=x_{i}$. First, we analyze the case of the unique equilibrium being an inner point. Assume that the trajectory of the system hits the boundary $\tilde{d}=\tilde{d}_{\max }:=d_{\max }-d^{*}>0$. In order for the trajectory to stay on this boundary, we need $\dot{\tilde{d}}=\tilde{x} / C \geq 0$. However, we have $\dot{\tilde{x}}<0$ from (8) as due to (9) $g(\tilde{x})>0$ when $\tilde{x}<0$. Then $\tilde{x}$, and hence $\dot{\tilde{d}}$, necessarily become negative after some time. Thus, the trajectory has to leave this boundary. Furthermore, we have $\dot{V} \leq 0$ on the trajectory of the system. As a result, once the trajectory leaves a boundary it can never hit it again.

We proceed with other three boundaries in a similar fashion. Assume that the trajectory of the system hits the boundary $\tilde{d}=\tilde{d}_{\min }:=-d^{*}<0$. Since from (8) and (9) $\dot{\tilde{x}}>0, \tilde{x}$ and $\dot{\tilde{d}}$ necessarily become positive after some time. Hence, the trajectory has to leave the boundary. On the other hand, when $\tilde{x}=x_{\max }-x^{*}$, we have $g(\tilde{x})<0$ and $\dot{\tilde{d}}>0$. Thus, we obtain $\tilde{d}>0$ after some delay and $\dot{\tilde{x}}<0$ from (8), forcing the trajectory out of the boundary. Finally, in the case of $\tilde{x}=$ $-x^{*}<0$ we have $g(\tilde{x})>0$ and $\dot{\tilde{d}}<0$. Thus, after some time $\tilde{d}<0$, and hence $\tilde{\tilde{x}}>0$ from (8). Again, the trajectory leaves the boundary and never returns back due to the non-increasing Lyapunov function $V$.

In the case of a boundary solution, once the trajectory reaches the equilibrium point it stays on the boundary. For example, assume that $x^{*}=x_{\max }<C$. Then, from (6) we have $\dot{x}=\dot{\tilde{x}}>0$. Furthermore, $\dot{\tilde{d}}=0$ from (8). Thus, the trajectory stays on the boundary and at the equilibrium point. In conclusion, the system (7) with boundaries given in (4) and (6) is globally asymptotically stable on the set $\Omega:=\left\{(x, d) \in \mathbb{R}^{2}: 0 \leq x \leq x_{\max }\right.$ and $\left.0 \leq d \leq d_{\max }\right\}$ by LaSalle's invariance theorem [18].

\section{B. Stability for a Single Link with Multiple Users}

The analysis for a single link with multiple users is a fairly straightforward generalization of the single-link singleuser case discussed above. The system has again a unique equilibrium point $\left(\mathbf{x}^{*}, d^{*}\right)^{2}$, at which $\left(1 / \alpha_{i}\right) d U_{i}\left(x_{i}^{*}\right) / d x_{i}$ is

\footnotetext{
${ }^{2}$ The proof of uniqueness for a more general case which also captures this special case will be provided in Proposition III.1
} 
independent of $i, \bar{x}^{*}=C$ and $d^{*}=\left(1 / \alpha_{i}\right) d U_{i}\left(x_{i}^{*}\right) / d x_{i}$. Defining the system around this equilibrium point as in (8), we obtain

$$
\begin{aligned}
& \dot{\tilde{x}}_{i}(t)=g_{i}\left(\tilde{x}_{i}\right)-\alpha_{i} \tilde{d}(t), i=1, \ldots, M, \\
& \dot{\tilde{d}}(t)=\frac{1}{C} \sum_{i=1}^{M} \tilde{x}_{i},
\end{aligned}
$$

where $\alpha:=\left[\alpha_{1}, \ldots, \alpha_{M}\right]$ is the user pricing vector, $U_{1}\left(x_{1}\right), \ldots, U_{M}\left(x_{M}\right)$ are strictly concave user utility functions, and the functions $g_{i}\left(x_{i}\right)$ are defined similarly as in the case of (9).

Let us define the generalized set $\tilde{\Omega}$ as

$$
\begin{array}{r}
\tilde{\Omega}=\left\{(\tilde{\mathbf{x}}, \tilde{d}) \in \mathbb{R}^{M+1}:-x_{i}^{*} \leq \tilde{x}_{i} \leq x_{i, \max }-x_{i}^{*}, \forall i\right. \\
\text { and } \left.-d^{*} \leq \tilde{d} \leq d_{\max }-d^{*}\right\},
\end{array}
$$

where $d_{\max }$ and $x_{i, \max }$ are upper-bounds on $d$ and $x_{i}$ respectively.

We next define a Lyapunov function on the set $\tilde{\Omega}$, similar to the one of (11):

$$
V(\tilde{\mathbf{x}}, \tilde{d})=\sum_{i=1}^{M} \frac{1}{\alpha_{i}}\left(\tilde{x}_{i}\right)^{2}+C(\tilde{d})^{2} .
$$

The rest of the analysis is similar to the one in the case of a single link with a single user, and therefore it will not be carried out. In particular, $\dot{V}=\sum_{i=1}^{M} \frac{2}{\alpha_{i}} g_{i}\left(\tilde{x}_{i}\right) \tilde{x}_{i} \leq 0$, and is equal to zero only if $\tilde{x}_{i}=0 \forall i \Rightarrow \tilde{d}=0$. Again, the system is globally asymptotically stable.

\section{Stability for a General Network Topology with Multiple Users}

We finally establish the stability of the system under a general network topology with multiple links, and with a general routing matrix $\mathbf{A}$ as defined in (1). The generalized system is described by (again without the boundary effects)

$$
\begin{aligned}
& \dot{x}_{i}(t)=\frac{d U_{i}\left(x_{i}\right)}{d x_{i}}-\alpha_{i} D_{i}(\mathbf{x}, t), i=1, \ldots, M, \\
& \dot{d}_{l}(t)=\frac{\bar{x}_{l}}{C_{l}}-1, l=1, \ldots, L,
\end{aligned}
$$

where $D_{i}(\mathbf{x}, t)=\sum_{l \in R_{i}} d_{l}(\mathbf{x}, t), \bar{x}_{l}:=\sum_{i: l \in R_{i}} x_{i}$, and $C_{l}$ is the capacity of the $l^{t h}$ link. For this general case, equilibrium point or points of the system cannot be described explicitly. Therefore, we first investigate the uniqueness of the equilibrium. Toward this end, we assume that $\mathbf{A}$ is a full row rank matrix with $M \geq L$ which is in fact no loss of generality as non-bottleneck links on the network have no effect on the equilibrium point, and can be safely left out.

Proposition III.1. When A is full row rank, the system (15) has a unique equilibrium point.

Proof. By setting $\dot{x}_{i}(t)$ and $\dot{d}_{l}(t)$ equal to zero for all $l$ and $i$ one obtains

$$
\begin{gathered}
\mathbf{A} \mathbf{x}=\mathbf{C} \\
\mathbf{f}(\alpha, \mathbf{x})=\mathbf{A}^{T} \mathbf{d},
\end{gathered}
$$

where $\mathbf{d}=\left[d_{1}, \ldots, d_{L}\right]$ is the delay vector at the links, and the nonlinear vector function $\mathbf{f}$ is defined as

$$
\mathbf{f}(\alpha, \mathbf{x}):=\left[\frac{1}{\alpha_{i}} \frac{d U_{i}}{d x_{i}}, \ldots, \frac{1}{\alpha_{M}} \frac{d U_{M}}{d x_{M}}\right] .
$$

Suppose that there are two different equilibrium points $\left(\mathbf{x}_{1}^{*}, \mathbf{d}_{1}^{*}\right)$ and $\left(\mathbf{x}_{2}^{*}, \mathbf{d}_{2}^{*}\right)$. Then, from (16) it follows that

$$
\mathbf{A}\left(\mathbf{x}_{1}^{*}-\mathbf{x}_{2}^{*}\right)=0 \Leftrightarrow\left(\mathbf{x}_{1}^{*}-\mathbf{x}_{2}^{*}\right)^{T} \mathbf{A}^{T}=0
$$

Similarly, from (17) we have

$$
\mathbf{f}\left(\alpha, \mathbf{x}_{1}^{*}\right)-\mathbf{f}\left(\alpha, \mathbf{x}_{2}^{*}\right)=\mathbf{A}^{T}\left(\mathbf{d}_{1}^{*}-\mathbf{d}_{2}^{*}\right) .
$$

Multiplying this with $\left(\mathbf{x}_{1}^{*}-\mathbf{x}_{2}^{*}\right)^{T}$ from left we obtain

$$
\left(\mathbf{x}_{1}^{*}-\mathbf{x}_{2}^{*}\right)^{T}\left[\mathbf{f}\left(\alpha, \mathbf{x}_{1}^{*}\right)-\mathbf{f}\left(\alpha, \mathbf{x}_{2}^{*}\right)\right]=0
$$

We rewrite this as

$$
\sum_{i=1}^{M}\left(\mathbf{x}_{1 i}^{*}-\mathbf{x}_{2 i}^{*}\right)^{T} \frac{1}{\alpha_{i}}\left[\frac{d U_{i}\left(x_{1 i}^{*}\right)}{d x_{i}}-\frac{d U_{i}\left(x_{2 i}^{*}\right)}{d x_{i}}\right]=0 .
$$

Since $U_{i}$ 's are strictly concave, each term in the summation is negative, with equality holding only if $x_{1 i}^{*}=x_{2 i}^{*}$. Hence, we conclude that $x^{*}$ has to be unique, that is

$$
\mathbf{x}^{*}=\mathbf{x}_{1}^{*}=\mathbf{x}_{2}^{*}
$$

From this, and (15), it immediately follows that $D_{i}, i=$ $1, \ldots, M$, are unique. This does not however immediately imply that $d_{l}, l=1, \ldots, L$, are also unique. To establish this, we first multiply (17) from left by $\mathbf{A}$ :

$$
\mathbf{A} \mathbf{f}\left(\alpha, \mathbf{x}^{*}\right)=\mathbf{A} \mathbf{A}^{T} \mathbf{d}
$$

Since $\mathbf{A}$ is of full row rank, the square matrix $\mathbf{A} \mathbf{A}^{T}$ is full rank, and hence invertible. Thus, we obtain a unique $\mathbf{d}^{*}$ for a given equilibrium flow vector $\mathrm{x}^{*}$ :

$$
\mathbf{d}^{*}=\left(\mathbf{A} \mathbf{A}^{T}\right)^{-1} \mathbf{A f}\left(\alpha, \mathbf{x}^{*}\right)
$$

As a result, $\left(\mathrm{x}^{*}, \mathbf{d}^{*}\right)$ constitutes a unique equilibrium point for the system (15).

We note that the unique equilibrium point of the system is only an approximation to the Nash equilibrium since the effect of the $i^{t h}$ user on the delay, $D_{i}(\mathbf{x}, t)$, s/he experiences has been ignored. This approximation becomes more accurate as the number of users in the network increases. In addition, we again assume that $\alpha$ is chosen in such a way that the equilibrium point is an inner solution, $\mathbf{d}^{*}<\mathbf{d}_{\max }$.

Defining the delays at links, $d_{l}$, and user flow rates, $x_{i}$, around the equilibrium as $\tilde{d}_{l}:=d_{l}-d_{l}^{*}$ and $\tilde{x}_{i}:=x_{i}-x_{i}^{*}$, respectively, for all $l$ and $i$, we obtain the following system around the equilibrium:

$$
\begin{aligned}
\dot{\tilde{x}}_{i}(t) & =g_{i}\left(\tilde{x}_{i}\right)-\alpha_{i} \tilde{D}_{i}(t), i=1, \ldots, M, \\
\dot{\tilde{d}}_{l}(t) & =\frac{1}{C_{l}} \sum_{i: l \in R_{i}} \tilde{x}_{i}, l=1, \ldots, L,
\end{aligned}
$$

where $\tilde{D}_{i}=\sum_{l \in R_{i}} \tilde{d}_{l}$, and $g_{i}($.$) is defined as in (9). For the$ time being, we ignore the effect of boundaries on the system. 
Let us define a set $\tilde{\Omega}$ (as before) as

$$
\begin{array}{r}
\tilde{\Omega}=\left\{(\tilde{\mathbf{x}}, \tilde{\mathbf{d}}) \in \mathbb{R}^{M+L}:-x_{i}^{*} \leq \tilde{x}_{i} \leq x_{i, \max }-x_{i}^{*}\right. \\
\text { and } \left.-d_{l}^{*} \leq \tilde{d}_{l} \leq d_{l, \text { max }}-d_{l}^{*}, \forall i, l\right\},
\end{array}
$$

where $d_{l, \max }$ and $x_{i, \max }$ are upper bounds on $d_{l}$ and $x_{i}$, respectively.

We next define a Lyapunov function on the set $\tilde{\Omega}$ as a generalized version of the one of (14):

$$
V(\tilde{\mathbf{x}}, \tilde{\mathbf{d}})=\sum_{i=1}^{M} \frac{1}{\alpha_{i}}\left(\tilde{x}_{i}\right)^{2}+\sum_{l=1}^{L} C_{l}\left(\tilde{d}_{l}\right)^{2}
$$

The function $V(\tilde{x}, \tilde{d})$ is positive definite on $\tilde{\Omega}$, and its derivative along the system trajectories is given by

$$
\dot{V}(\tilde{\mathbf{x}}, \tilde{\mathbf{d}})=\sum_{i=1}^{M} \frac{2}{\alpha_{i}} g_{i}\left(\tilde{x}_{i}\right) \tilde{x}_{i} \leq 0,
$$

where the inequality follows because $g_{i}\left(\tilde{x}_{i}\right) \tilde{x}_{i} \leq 0 \forall i$. Thus, $\dot{V}(\tilde{\mathbf{x}}, \tilde{\mathbf{d}})$ is negative semidefinite. Let $S:=\{(\tilde{\mathbf{x}}, \tilde{\mathbf{d}}) \in \tilde{\Omega}$ : $\dot{V}(\tilde{\mathbf{x}}, \tilde{\mathbf{d}})=0\}$. It follows as before that $S=\{(\tilde{\mathbf{x}}, \tilde{\mathbf{d}}) \in \tilde{\Omega}$ : $\tilde{\mathbf{x}}=0\}$. Hence, for any trajectory of the system that belongs identically to the set $S$, we have $\tilde{\mathbf{x}}=0$. It follows directly from (18) that

$$
\begin{aligned}
\tilde{\mathbf{x}}=0 & \Rightarrow \dot{\tilde{\mathbf{x}}}=0 \Rightarrow g_{i}(\tilde{x})=0 \forall i \\
& \Rightarrow \tilde{D}_{i}=0 \forall i \Rightarrow \tilde{d}_{l}=0 \forall l,
\end{aligned}
$$

where the last line is due to the fact that $\tilde{D}=\mathbf{A}^{T} \tilde{\mathbf{d}}^{*}$ and the matrix $\mathbf{A}$ is of full row rank. Therefore, the only solution that can stay identically in $S$ is the zero solution, which corresponds to the unique equilibrium of the original system.

We now investigate the effect of the boundaries given in $\tilde{\Omega}$ and described by (4) and (6). First, we analyze the case when the unique equilibrium is not on the boundaries of the set $\tilde{\Omega}$. Consider the case where $\tilde{d}_{\hat{l}}=d_{\hat{l}, \text { max }}-d_{\hat{l}}^{*}$ for some link $l=\hat{l}$ while all links except $\hat{l}$ are in equilibrium. Then, for any user $i$ whose flow passes through link $\hat{l}$, and $\tilde{x}_{i}>0$, we have $g_{i}\left(\tilde{x}_{i}\right)<0$, and from (18) $\dot{\tilde{x}}_{i}<0$. Therefore, $\sum_{i: \hat{l} \in R_{i}} \tilde{x}_{i}$ decreases until it is negative, which in turn makes $\dot{\tilde{d}}_{\hat{l}}<0$. Thus, the trajectory leaves the boundary. Since $\dot{V} \leq 0$, the trajectory cannot hit the same boundary again. The case $\tilde{d}_{l}=$ $-d_{l}^{*}$ can be handled in a similar fashion. We note that, the case $\tilde{d}_{l}=-d_{l}^{*}$, if it occurs in equilibrium, corresponds to an empty buffer at the link $l$, where the link has no effect on the system for the given set of parameters. As a result, that link can be left out.

For the boundary at $\tilde{x}_{i}=x_{i, \max }-x_{i}^{*}$, we have $D_{i}>0$ given that all other users passing through links on the path of the $i^{\text {th }}$ user are in equilibrium. Then, it immediately follows from (18) that $\dot{\tilde{x}}_{i}<0$ and the trajectory leaves the boundary for good. Otherwise, we have a boundary solution with a subset of users transmitting with maximum feasible flow rate, $\tilde{x}_{i, \max }$, which contradicts with the initial hypothesis on the equilibrium point. A similar argument holds for the case $\tilde{x}_{i}=-x_{i}^{*}$, i.e., either there is a boundary solution or the trajectory eventually leaves the boundary and does not hit it again due to the Lyapunov analysis.

We next analyze the case of the equilibrium being on the boundary. Similar to the single user case, once the trajectory reaches the equilibrium point it stays on the boundary. Consider the case where $x_{i}^{*}=x_{i, \max }$ for the $i^{t h}$ user, while other users have equilibrium flows that are less than the maximum. Then, from (6) we have $\dot{x}_{i}=\dot{\tilde{x}}_{i}>0$. Furthermore, it follows from (18) that $\dot{d}_{l}=0, \forall l$. Thus, the trajectory stays on the boundary. We note that the other cases can be handled in a similar fashion. These results are summarized in the following theorem, where we again invoke LaSalle's invariance theorem:

Theorem III.2. Let A be full row rank. The system

$$
\begin{aligned}
& \dot{x}_{i}(t)=\frac{d U_{i}\left(x_{i}\right)}{d x_{i}}-\alpha_{i} D_{i}(\mathbf{x}, t), i=1, \ldots, M, \\
& \dot{d}_{l}(t)=\frac{\bar{x}_{l}}{C_{l}}-1, l=1, \ldots, L,
\end{aligned}
$$

with the unique equilibrium point $\left(\mathrm{x}^{*}, \mathrm{~d}^{*}\right)$, and boundary point behavior described by (4) and (6), is globally asymptotically stable on the set

$$
\begin{array}{r}
\Omega:=\left\{(x, \mathbf{d}) \in \mathbb{R}^{M+L}: 0 \leq x_{i} \leq x_{i, \max }\right. \\
\text { and } \left.0 \leq d_{l} \leq \bar{d}_{l, \max }, \forall i, l\right\} .
\end{array}
$$

\section{Stability Under Information DElay}

It was shown in Section III that the system described by (4) and (6) is globally asymptotically stable under a general network topology. We now investigate the global stability of the system under arbitrary propagation delays, which we are also going to refer as information delay, and denote by $r$. First, we analyze the simple case of a single link with a single user to gain insight into the problem. Next, we generalize the analysis to a general network with a single bottleneck node and multiple users.

\section{A. Stability for a Single Link with a Single User under Information Delay}

For the case of a single user on a single link, we modify equation (7) describing the system around the equilibrium by introducing a maximum propagation (information) delay $r$ between the user and the link, which we assume to be a constant :

$$
\begin{aligned}
& \dot{\tilde{x}}(t)=g(\tilde{x})-\alpha \tilde{d}(t-r) \\
& \dot{\tilde{d}}(t)=\frac{1}{C} \tilde{x}(t-r)
\end{aligned}
$$

Notice that (21) is a set of delay differential equations. Such systems have been studied extensively in [19], [20]. Here we will particularly make use of the methods presented in Chapter 4.2 of [20]. From (21), we immediately have

$$
\dot{\tilde{x}}(t)=g(\tilde{x}(t))-\alpha \tilde{d}(t+r)+\alpha[\tilde{d}(t+r)-\tilde{d}(t-r)]
$$

and

$$
\dot{\tilde{x}}(t-r)=g(\tilde{x}(t-r))-\alpha \tilde{d}(t)+\frac{\alpha}{C} \int_{-2 r}^{0} \tilde{x}(t+s) d s .
$$


On the set $\tilde{\Omega}$ defined by (10), we define a Lyapunov function

$$
\begin{aligned}
V(\tilde{x}, \tilde{d})= & \frac{1}{\alpha}(\tilde{x}(t-r))^{2}+C(\tilde{d}(t))^{2} \\
& +\frac{1}{C} \int_{-2 r}^{0} \int_{t+s}^{t} \tilde{x}^{2}(u-r) d u d s,
\end{aligned}
$$

which is positive definite. Taking the derivative of $V$ along the system trajectories, we obtain

$$
\begin{aligned}
\dot{V}(\tilde{x}, \tilde{d})= & \frac{2}{\alpha} g(\tilde{x}(t-r)) \tilde{x}(t-r) \\
& +\frac{2}{C} \int_{-2 r}^{0} \tilde{x}(t-r) \tilde{x}(t+s-r) d s \\
& +\frac{1}{C} \int_{-2 r}^{0}\left[\tilde{x}^{2}(t-r)-\tilde{x}^{2}(t+s-r)\right] d s
\end{aligned}
$$

Using the simple algebraic inequality

$$
2 \tilde{x}(t-r) \tilde{x}(t+s-r) \leq \tilde{x}^{2}(t-r)+\tilde{x}^{2}(t+s-r),
$$

one can bound the derivative $\dot{V}$ above by

$$
\dot{V}(\tilde{x}, \tilde{d}) \leq \frac{2}{\alpha} g(\tilde{x}(t-r)) \tilde{x}(t-r)+\frac{4 r}{C} \tilde{x}^{2}(t-r)
$$

Thus, $\dot{V}(\tilde{x}, \tilde{d})$ can be made negative semi-definite by imposing a condition on the maximum delay $r$. In this case, let $S:=\{(\tilde{x}, \tilde{d}) \in \tilde{\Omega}: \dot{V}(\tilde{x}, \tilde{d})=0\}$. It follows from (9) that $S=\{(\tilde{x}, \tilde{d}) \in \tilde{\Omega}: \tilde{x}=0\}$. Hence, for any trajectory of the system that belongs to $S$, we have $\tilde{x} \equiv 0$. It also follows directly from (21) that

$$
\tilde{x} \equiv 0 \Rightarrow \dot{\tilde{x}}=0 \Rightarrow g(\tilde{x})=0 \Rightarrow \tilde{d}=0 .
$$

Therefore, the only solution that can stay identically in $S$ is the zero solution, which corresponds to the unique equilibrium of the original system (7).

We thus conclude that the system (21) is asymptotically stable by LaSalle's invariance theorem if the maximum delay $r$ satisfies the condition

$$
r<\frac{C}{2 \alpha} k,
$$

where $k$ is defined as

$$
k:=\inf _{-x^{*} \leq \tilde{x} \leq x_{\max }-x^{*}}\left|\frac{g(\tilde{x})}{\tilde{x}}\right| .
$$

In order to gain further insight into this condition, we compute the parameter $k$ for the specific case when the utility function is taken as the logarithmic one, that is $U(x)=u \log (x+1)$. In this case we obtain

$$
g(\tilde{x})=\frac{u}{x+1}-\frac{u}{x^{*}+1}=\frac{-u \tilde{x}}{(x+1)\left(x^{*}+1\right)}
$$

and hence

$$
k=\min _{0 \leq x \leq x_{\max }} \frac{u}{(x+1)\left(x^{*}+1\right)}=\frac{u}{\left(x_{\max }+1\right)\left(x^{*}+1\right)} .
$$

The requirement on the delay term $r$ is dependent on the equilibrium $x^{*}$, and since $x^{*} \in\left[0, x_{\max }\right]$, a safe bound on $r$ is

$$
r<\frac{u C}{2 \alpha\left(x_{\max }+1\right)^{2}} \text {. }
$$

Of course a better bound can be obtained on $r$ if we know that $x^{*} \ll x_{\max }$, and that the trajectory also remains in a small neighborhood of the equilibrium, $x^{*}$. This would very much be dependent on the application at hand.

The analysis of the effect of boundaries on the system is almost identical to the one of the case without delay. Assume that $\tilde{d}(t)=\tilde{d}_{\text {max }} \forall t \in[-r, 0]$. In order for the trajectory to stay on the boundary at $t>0$, one needs $\tilde{x}(t)>0 \forall t \in$ $[-r, 0]$. However, we have $\dot{\tilde{x}}(t)<0$ from (21). Hence, after some time, $\dot{\tilde{d}}<0$, and the trajectory leaves the boundary. Since $\dot{V}<0$ the system trajectory may never return to the boundary. The analysis of the remaining boundaries can be handled in a similar way, and will be omitted. This now brings us to the following theorem, where again LaSalle's invariance theorem is invoked:

\section{Theorem IV.1. The system}

$$
\begin{aligned}
& \dot{x}(t)=\frac{d U(x(t))}{d x}-\alpha d(t-r) \\
& \dot{d}(t)=\frac{1}{C} x(t-r)-1,
\end{aligned}
$$

with the unique equilibrium point $\left(\mathbf{x}^{*}, \mathbf{d}^{*}\right)$ and boundary point behavior described by (4) and (6) is globally asymptotically stable on the set $\Omega$ if the maximum delay, $r$, in the system satisfies the condition

$$
r<\frac{k C}{2 \alpha},
$$

where $k:=\inf _{-x^{*} \leq \tilde{x} \leq x_{\max }-x^{*}}|g(\tilde{x}) / \tilde{x}|$.

\section{B. Stability for a Single (Bottleneck) Link with Multiple Users under Information Delay}

We now generalize the preceding analysis of a single link with a single user to multiple users by introducing user specific maximum propagation delays $r=\left[r_{1}, \ldots, r_{M}\right]$ between the link and the users. The system has a unique equilibrium point $\left(\mathbf{x}^{*}, d^{*}\right)$ as characterized in Section III. Modifying the system equations (12) around this equilibrium point by introducing the associated maximum propagation delays, we obtain

$$
\begin{aligned}
& \dot{\tilde{x}}_{i}(t)=g_{i}\left(\tilde{x}_{i}(t)\right)-\alpha_{i} \tilde{d}\left(t-r_{i}\right), i=1, \ldots, M \\
& \dot{\tilde{d}}(t)=\frac{1}{C} \sum_{i=1}^{M} \tilde{x}_{i}\left(t-r_{i}\right)
\end{aligned}
$$

Following an approach similar to the one in the single user case with delay, one gets for the $i^{\text {th }}$ user

$$
\begin{aligned}
\dot{\tilde{x}}_{i}\left(t-r_{i}\right)= & g_{i}\left(\tilde{x}_{i}\left(t-r_{i}\right)\right)-\alpha_{i} \tilde{d}(t) \\
& +\frac{\alpha_{i}}{C} \int_{-2 r_{i}}^{0} \sum_{j=1}^{M} \tilde{x}_{j}\left(t+s-r_{j}\right) d s .
\end{aligned}
$$

We again define a positive definite Lyapunov function on the corresponding set $\tilde{\Omega}$ defined by (13) :

$$
\begin{aligned}
V(\tilde{\mathbf{x}}, \tilde{d})= & \sum_{i=1}^{M} \frac{1}{\alpha_{i}}\left(\tilde{x}_{i}\left(t-r_{i}\right)\right)^{2}+C(\tilde{d}(t))^{2} \\
& +\frac{M}{C} \sum_{i=1}^{M} \int_{-2 r_{i}}^{0} \int_{t+s}^{t} \tilde{x}_{i}^{2}\left(u-r_{i}\right) d u d s .
\end{aligned}
$$


Taking the derivative of $V$ along the system trajectories, we obtain

$$
\begin{aligned}
& \dot{V}(\tilde{\mathbf{x}}, \tilde{d})=\sum_{i=1}^{M} \frac{2}{\alpha_{i}} g_{i}\left(\tilde{x}_{i}\left(t-r_{i}\right)\right) \tilde{x}\left(t-r_{i}\right) \\
& +\frac{1}{C} \int_{-2 r_{i}}^{0} \sum_{i=1}^{M} \sum_{j=1}^{M} 2 \tilde{x}_{i}\left(t-r_{i}\right) \tilde{x}_{j}\left(t+s-r_{j}\right) d s \\
& +\frac{M}{C} \sum_{i=1}^{M} \int_{-2 r_{i}}^{0}\left[\tilde{x}_{i}^{2}(t-r)-\tilde{x}_{i}^{2}(t+s-r)\right] d s
\end{aligned}
$$

We bound the derivative $\dot{V}$ above by

$$
\begin{aligned}
\dot{V}(\tilde{\mathbf{x}}, \tilde{d}) \leq & \sum_{i=1}^{M} \frac{2}{\alpha_{i}} g_{i}\left(\tilde{x}_{i}\left(t-r_{i}\right)\right) \tilde{x}_{i}\left(t-r_{i}\right) \\
& +\frac{4 M r_{i}}{C} \tilde{x}_{i}^{2}\left(t-r_{i}\right)
\end{aligned}
$$

The derivative of $V$ can be made strictly negative by imposing a condition on the maximum delay in the system, $r_{\max }:=$ $\max _{i} r_{i}$. In this case, let $S:=\{(\tilde{\mathbf{x}}, \tilde{d}) \in \tilde{\Omega}: \dot{V}(\tilde{\mathbf{x}}, \tilde{d})=0\}$. It follows as before that $S=\{(\tilde{\mathbf{x}}, \tilde{d}) \in \tilde{\Omega}: \tilde{\mathbf{x}}=0\}$. Hence, for any trajectory of the system that belongs identically to the set $S$, we have $\tilde{\mathbf{x}}=0$. It also follows directly from (24) that

$$
\tilde{\mathbf{x}}=0 \Rightarrow \dot{\tilde{\mathbf{x}}}=0 \Rightarrow g_{i}(\tilde{x})=0 \forall i \Rightarrow \tilde{d}=0,
$$

where we have made use of the fact that the matrix $\mathbf{A}$ is of full row rank. Therefore, the only solution that can stay identically in $S$ is the zero solution, which corresponds to the unique equilibrium of the original system. As a result, the system (24) is asymptotically stable by LaSalle's invariance theorem if the maximum delay in the system, $r_{\max }$, satisfies the condition

$$
r_{\max }<\frac{k_{\min } C}{2 M \alpha_{\max }},
$$

where $\alpha_{\max }$ and $k_{\min }$ are defined as

$$
\begin{aligned}
& \alpha_{\max }:=\max _{i} \alpha_{i} \\
& k_{\min }:=\min _{i} \inf _{-x_{i}^{*} \leq \tilde{x}_{i} \leq x_{i, \max }-x_{i}^{*}}\left|\frac{g\left(\tilde{x}_{i}\right)}{\tilde{x}_{i}}\right|
\end{aligned}
$$

Notice that the bound on the maximum delay required for the stability in the system is affected by, among other things, the maximum pricing parameter and the number of users.

We next investigate the effect of boundaries on the system, first in the case of the equilibrium being an inner one. Consider the case $\tilde{d}(t)=d_{\max }-d^{*} \forall t \in[-r, 0]$. Then, for any user $i$ with $\tilde{x}_{i}(t)>0$, we have $g_{i}\left(\tilde{x}_{i}(t)\right)<0$, and from (24) $\dot{\tilde{x}}_{i}(t)<$ $0, t>0$. Therefore, $\sum_{i} \tilde{x}_{i}$ decreases until it is negative, which in turn makes $\dot{\tilde{d}}<0$. Thus, the trajectory leaves the boundary. Since $\dot{V}<0$, the trajectory cannot hit the same boundary again. A similar analysis also applies to the case $\tilde{d}=-d^{*}$. For boundaries on $\tilde{x}_{i}$, assume that all users but the $i^{\text {th }}$ one are in equilibrium, and $\tilde{x}_{i}=x_{i, \max }-x_{i}^{*} \forall t \in[-r, 0]$. Then, we necessarily have $\tilde{d}>0$ after some time, and hence $\dot{\tilde{x}}_{i}<0$. Thus, the trajectory leaves the boundary, and never returns due to the strictly decreasing nature of the Lyapunov function $V$.
Similar arguments also apply to the case when all users but the $i^{t h}$ one are in equilibrium, and $\tilde{x}_{i}=-x_{i}^{*}$. In the case of boundary solutions, the analysis is identical to earlier ones and therefore will be omitted. The following theorem now extends the results of Theorem IV.1 to the multi-user case.

Theorem IV.2. The system

$$
\begin{aligned}
& \dot{x}_{i}(t)=\frac{d U_{i}\left(x_{i}(t)\right)}{d x_{i}}-\alpha_{i} d(\mathbf{x}, t-r), i=1, \ldots, M, \\
& \dot{d}(t)=\frac{1}{C} \sum_{i=1}^{M} x_{i}\left(t-r_{i}\right)-1,
\end{aligned}
$$

with the unique equilibrium point $\left(\mathrm{x}^{*}, d^{*}\right)$, and boundary point behavior described by (4) and (6), is globally asymptotically stable on the corresponding set $\Omega$ defined by (20), if the maximum delay, $r_{\max }$, in the system satisfies the condition

$$
r_{\max }<\frac{k_{\min } C}{2 M \alpha_{\max }},
$$

where $\alpha_{\max }$ and $k_{\min }$ are defined in (27).

\section{An IMPLEMENTATion of THE CONGESTION CONTROL SCHEME}

The user responses in Section II are based on a continuous time formulation. In reality, however, users update their flow rates only at discrete time instances corresponding to multiples of RTT. Hence, for implementation purposes, we discretize the reaction function of the $i^{t h}$ user, and normalize it with respect to the RTT of the user. In addition, we need a specific utility function in order to quantify the user response in (6). Logarithmic utility functions are widely used in the literature not only because they have nice properties like strict concavity but also because they adequately capture several important concepts economics, such as the law of diminishing returns. We choose the following utility function for $i^{t h}$ user:

$$
U_{i}\left(x_{i}\right)=u_{i} \log \left(x_{i}+1\right),
$$

where $u_{i}$ is a user specific utility parameter. The optimal user response is, therefore, a discretized version of (6), and is given by

$$
x_{i}(t+1)=\left[x_{i}(t)+\kappa_{i}\left[\frac{u_{i}}{x_{i}(t)+1}-\alpha_{i} \sum_{l \in R_{i}} d_{l}(t)\right]\right]^{+},
$$

where $\kappa_{i}$ is a (user specific) step-size constant.

The congestion control scheme characterized by the user response (28) is implemented in a Game (theory) Based Congestion Control (GBCC) protocol using the Network Simulator $2(n s-2)$ [21]. The simulator $n s-2$ is chosen because it provides both a realistic environment for testing the proposed congestion control scheme and a level of abstraction for easy implementation. GBCC is a simple window based protocol for best-effort data traffic. It is devised as an end-to-end sliding window protocol [22], where the sender side adjusts its window size according to the reaction function (28). For simplicity, receiver window size is chosen as one. We also implement a version with a simple slow start mechanism 
where the window size is increased by one per RTT until a packet loss is observed. We next provide an overview on the GBCC scheme by summarizing the sender and receiver side functionalities.

\section{A. GBCC Protocol}

As one of the goals of GBCC protocol is compatibility with existing protocols, most of the functionality is on the sender side. Specifically, the sender side has the following functions:

- The sender puts sequence number and time stamp into the packet header. It estimates RTT and base RTT, which is calculated as the minimum of the RTTs up to that point, by using the received acknowledgment (ack) packets. The estimation method for RTT is the same as the one in [23].

- If a double ack is received, i.e. the same packet is acknowledged twice by the receiver, then it retransmits the packages beginning from the last acknowledged packet number. We note that this go back $n$ scheme [22] is implemented for its simplicity. In fact, better mechanisms with receiver window size being larger than one do exist.

- The sender updates the window size according to (28) using the current value of queueing delay, which is taken as the difference between the current RTT and base RTT. The window size, $W$, is strictly positive.

- If no ack packet is received within, say $2 R T T$, then sender retransmits previous packets beginning from the last acknowledged one, and reduces the window size.

The receiver side, on the other hand, has the function of acknowledging received packets. If no packet is received for a specific time, say $4 R T T$, last received packet is acknowledged again.

\section{Simulations}

We simulate the proposed congestion control scheme, GBCC, on $n s-2$. The underlying protocol used for routing is the standard IP. Links and queues are chosen to be duplex and drop-tail, respectively. For simplicity, we fix the packet sizes to 1, 000 bytes. In most of the cases queueing delays on the links are much smaller than the propagation delays that we choose. Hence, RTT's are approximately equal to twice the propagation delays.

First, we simulate GBCC without a slow start mechanism in the simple single-user single-link case. The parameters in (28) are chosen as $\alpha=30$ and $u=10,000$. The buffer size is $50 \mathrm{~KB}$ and propagation delay on the link is varied from $5 \mathrm{~ms}$ to $25 \mathrm{~ms}$, and to $100 \mathrm{~ms}$. We observe in Figure 1 that as RTT gets too large, the system becomes unstable in accordance with the analysis in Section IV. Notice that it takes up to 7 seconds for the flow to reach its capacity in this simulation. Therefore, we use the slow start version of GBCC for the rest of the study.

We next explore the interaction between GBCC and TCP on a single bottleneck link with $10 \mathrm{~ms}$ propagation delay. GBCC is TCP-friendly [1] since its long-term rate does not exceed the one of the TCP flow as observed in Figure 2. The fluctuation in the first two seconds is due to the slow start mechanism which

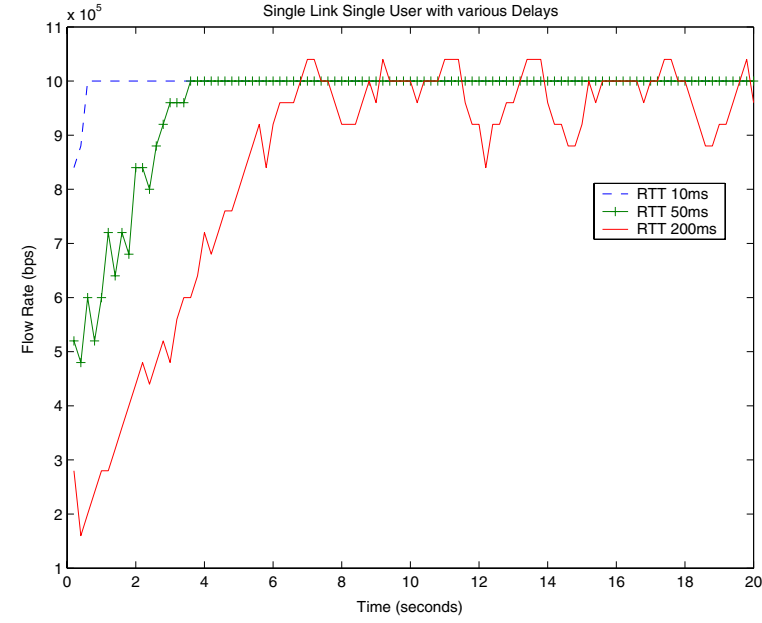

Fig. 1. A single user on a single link with $R T T=10,50$, and $200 \mathrm{~ms}$. This version of GBCC has no slow start mechanism.

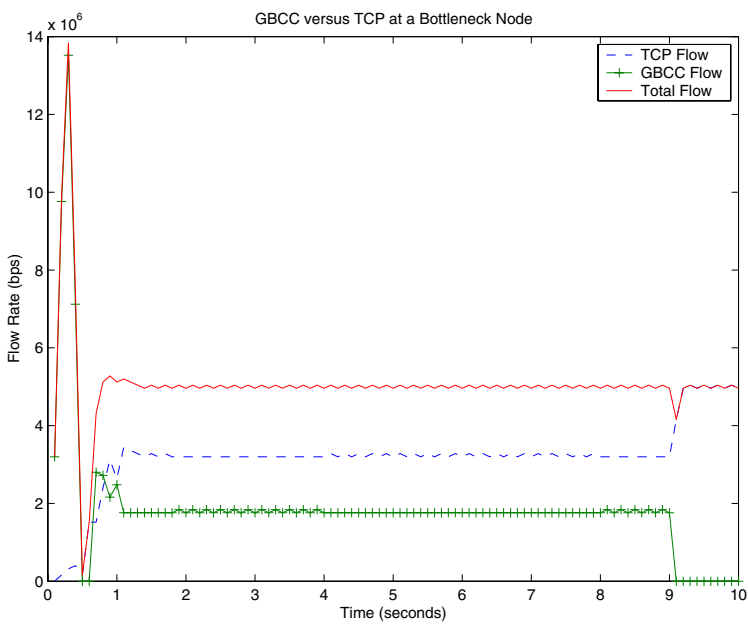

Fig. 2. GBCC flow versus TCP flow on a bottleneck link with $10 \mathrm{~ms}$ propagation delay.

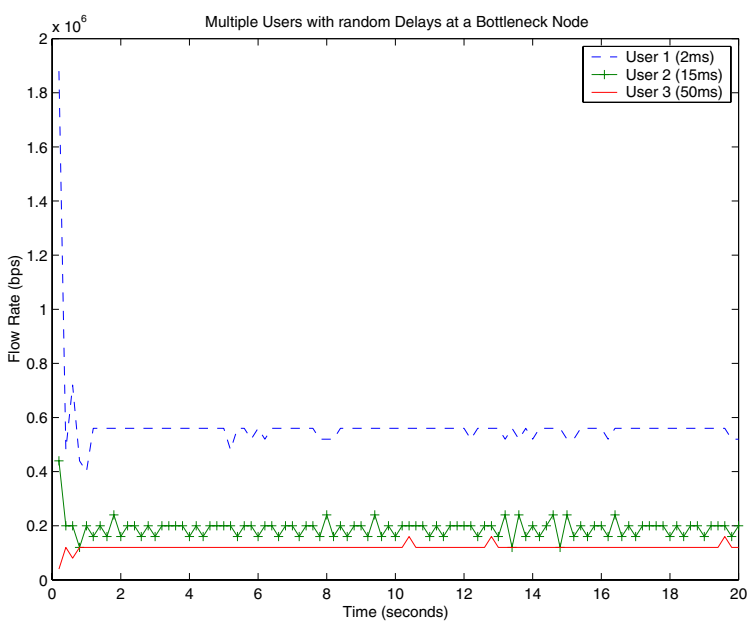

Fig. 3. Three out of 20 flows with various propagation delays $(2 \mathrm{~ms}, 15 \mathrm{~ms}$, and $50 \mathrm{~ms}$ ) sharing a $5 \mathrm{Mbps}$ bottleneck link. 
requires a packet loss for termination. In the final simulation on a single bottleneck link, there are 20 identical users with parameters $\alpha=50, u=400,000$, and propagation delays are randomly chosen between $2 \mathrm{~ms}$ and $50 \mathrm{~ms}$ according to a uniform distribution. We observe flows of 3 specific users with respective propagation delays of $2 \mathrm{~ms}, 15 \mathrm{~ms}$, and $50 \mathrm{~ms}$ in Figure 3. The system again converges to the equilibrium, however similar to TCP, GBCC favors flows with smaller RTT as it is a window-based scheme.

We then carry out a simulation with three users on a simple three node network topology with two $5 \mathrm{Mbps}$ links of $20 \mathrm{~ms}$ propagation delay as shown in Figure 4. While flows of users 1 and 2 pass through links 1 and 2 respectively, the flow of user 3 passes through both links. Cost parameters are chosen as $\alpha=30$ and $u=400,000$. User 3 is 'charged' more than others through summation of queueing delays as s/he uses resources on both links. Thus, having the same utility parameter as others, s/he obtains a smaller fraction of the bandwidth. Figure 5 depicts the flow rates of users 2 and 3, as observed in node 2 .

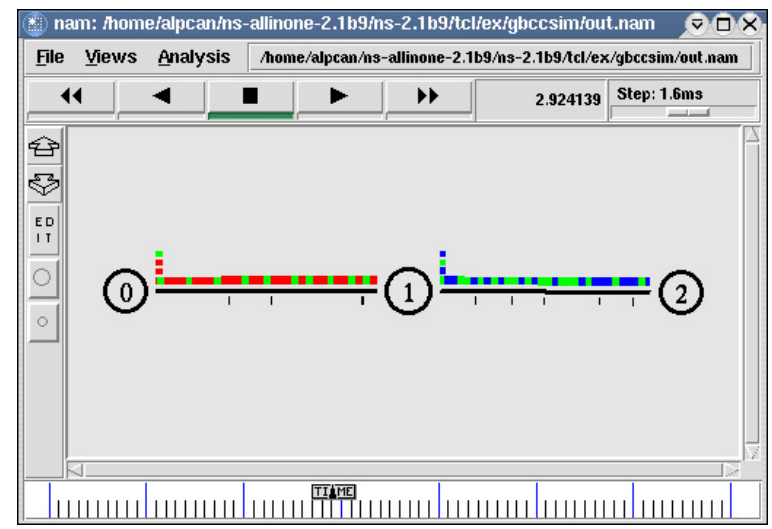

Fig. 4. A Nam screenshot of the simple network. Links are symmetric, and have a capacity of $5 \mathrm{Mbps}$ with $20 \mathrm{~ms}$ propagation delay.

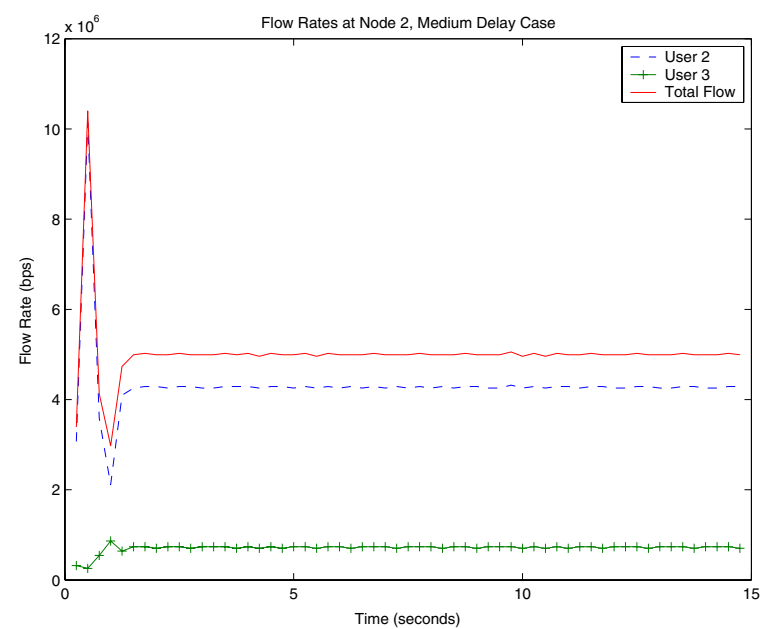

Fig. 5. Flows of users 2, 3, and total flow at node 2 are observed for 15 seconds.

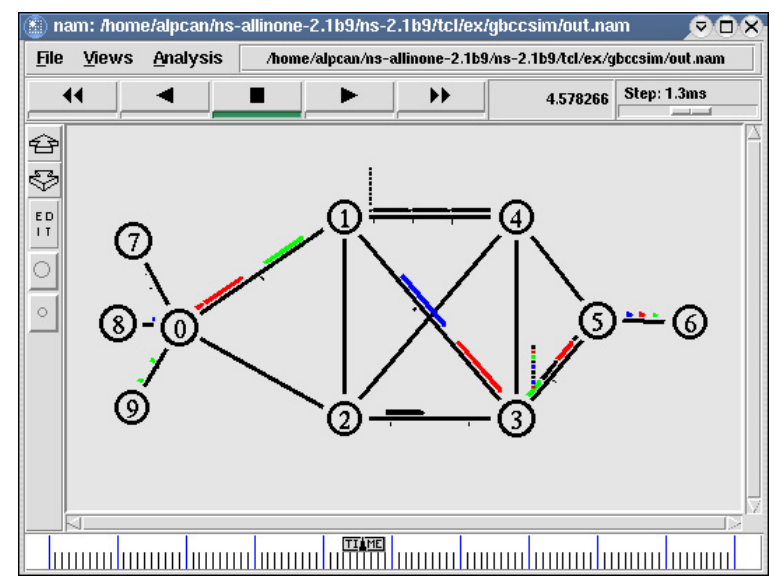

Fig. 6. A Nam screenshot of the general (arbitrary) topology network.

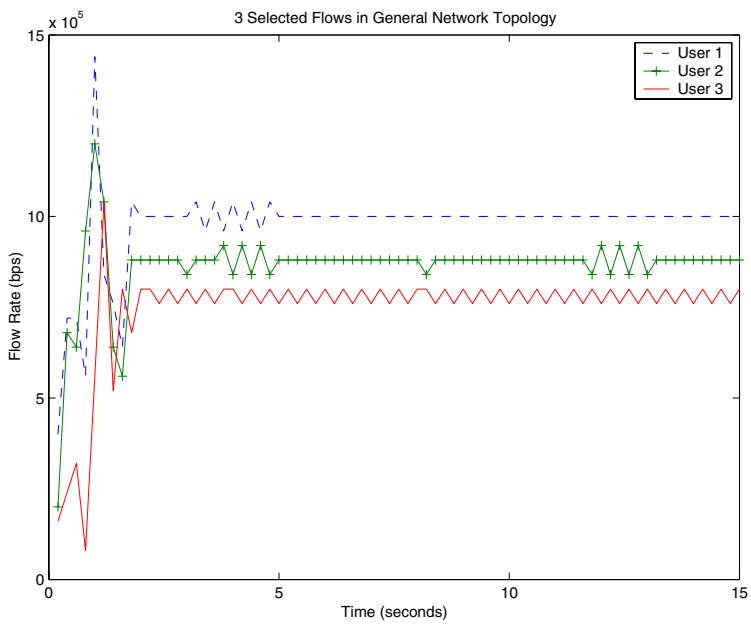

Fig. 7. Three flows from nodes 7,8 , and 9 to node 6 are shown where these users are symmetric and have the following cost parameters: $\alpha=30$ and $u=200,000$.

Finally, we simulate 10 users with various routes and experiencing various information delays on a seven node arbitrary topology network (Figure 6) with all links except the one between nodes 5 and 6 having capacity of $5 \mathrm{Mbps}$ each. The link between nodes 5 and 6 , on the other hand, has a capacity of $10 \mathrm{Mbps}$. The links have equal propagation delays of $5 \mathrm{~ms}$ each, except the links to nodes 7,8 , and 9, which have delays of $5 \mathrm{~ms}, 10 \mathrm{~ms}$, and $25 \mathrm{~ms}$, respectively. The users at nodes 7 , 8 , and 9 all have connections to node 6 and each experiences a different propagation delay. Figure 7 shows only the flows of these three users as measured at node 6 . We note that although the number of links in this simulation is equal to the number of users, the number of bottleneck links that affect the equilibrium flows is actually smaller. Hence, the routing matrix $\mathbf{A}$ is of full row rank. 


\section{CONCLUSION}

In this paper, we have developed and analyzed a congestion control game with a linear pricing scheme based on variations in the queueing delay experienced by the users. User demand for bandwidth is captured by a broad class of utility functions that are strictly increasing and strictly concave. The objective function for each user in this noncooperative game is defined as the difference between the pricing and utility functions. Using a network model based on fluid approximations, and through a realistic modeling of queues in the network, we have established the existence of a unique equilibrium, and the global stability of the equilibrium point for a general network topology. We have also provided sufficient conditions for system stability on a bottleneck link shared by multiple users under non-negligible propagation delays.

We have implemented and simulated a simple, windowbased, end-to-end congestion control scheme in $n s-2$ network simulator based on the theoretical foundations of the congestion control game. We have investigated several properties of the scheme developed through simulations on a single bottleneck link and on various general network topologies with non-negligible propagation delays. These simulations reveal that the implemented scheme not only confirms the theoretical results but is also TCP-friendly.

There still remain a few open issues and many directions for future research. For example, there is still ample room for improvement in the implementation of the congestion control scheme, such as increasing the receiver window size and fine tuning the slow start mechanism. Another topic for further study would be to devise a methodology for choosing the pricing parameter $\alpha$. Yet another direction for future research would be the derivation of improved (less restrictive) sufficient conditions on the maximum delay allowable in a general network, to ensure stability of the overall system.

\section{REFERENCES}

[1] S. Floyd and K. Fall, "Promoting the use of end-to-end congestion control in the internet," IEEE/ACM Transactions on Networking, vol. 7, no. 4, pp. 458 -472, August 1999. [Online]. Available: citeseer.nj.nec.com/article/floyd99promoting.html

[2] T. Başar and G. J. Olsder, Dynamic Noncooperative Game Theory. 2nd ed. Philadelphia, PA: SIAM, 1999.

[3] H. Yaiche, R. R. Mazumdar, and C. Rosenberg, "A game theoretic framework for bandwidth allocation and pricing in broadband networks," IEEE/ACM Transactions on Networking, vol. 8, pp. 667-678, October 2000.

[4] E. Altman, T. Başar, T. Jimenez, and N. Shimkin, "Competitive routing in networks with polynomial costs," IEEE Transactions on Automatic Control, vol. 47(1), pp. 92-96, January 2002.

[5] E. Altman and T. Başar, "Multi-user rate-based flow control," IEEE Transactions on Communications, vol. 46(7), pp. 940-949, july 1998.

[6] A. Orda, R. Rom, and N. Shimkin, "Competitive routing in multiuser communication networks," IEEE/ACM Transactions on Networking, vol. 1, pp. 510-521, October 1993.

[7] F. Kelly, A. Maulloo, and D. Tan, "Rate control in communication networks: shadow prices, proportional fairness and stability," Journal of the Operational Research Society, vol. 49, pp. 237-252, 1998.

[8] F. P. Kelly, "Charging and rate control for elastic traffic," European Transactions on Telecommunications, vol. 8, pp. 33-37, January 1997.

[9] R. Gibbens and P. Key, "Distributed control and resource marking using best-effort routers," IEEE Network, pp. 54-59, May 2001.
[10] S. Kunniyur and R. Srikant, "End-to-end congestion control schemes: Utility functions, random losses and ECN marks," in INFOCOM, 2000, pp. 1323-1332. [Online]. Available: citeseer.nj.nec.com/358188.html

[11] R. J. La and V. Anantharam, "Charge-sensitive TCP and rate control in the internet," in $\operatorname{INFOCOM}$ (3), 2000, pp. 1166-1175. [Online]. Available: citeseer.nj.nec.com/320096.html

[12] J. Mo and J. Walrand, "Fair end-to-end window-based congestion control," IEEE/ACM Transactions on Networking, vol. 8, p. 556567 , October 2000

[13] L. S. Brakmo and L. L. Peterson, "TCP vegas: End to end congestion avoidance on a global internet," IEEE Journal on Selected Areas in Communications, vol. 13, no. 8, pp. 1465-1480, 1995. [Online]. Available: citeseer.nj.nec.com/brakmo95tcp.html

[14] J. Mo, R. J. La, V. Anantharam, and J. C. Walrand, "Analysis and comparison of TCP reno and vegas," in Proc. IEEE Infocom, 1999, pp. 1556-1563. [Online]. Available: citeseer.nj.nec.com/331728.html

[15] J. Widmer, R. Denda, and M. Mauve, "A survey on TCP-friendly congestion control," IEEE Network, vol. 15, no. 3, pp. 28-37, 2001. [Online]. Available: citeseer.nj.nec.com/widmer01survey.html

[16] T. Alpcan and T. Başar, "A variable rate model with QoS guarantees for real-time internet traffic," in Proceedings of the SPIE Internat. Symp on Information Technologies, vol. 2411, November 2000.

[17] T. Başar and R. Srikant, "Revenue-maximizing pricing and capacity expansion in a many-users regime," in Proc. IEEE Infocom, New York, New York, June 23-27 2002, pp. 1556-1563.

[18] H. K. Khalil, Nonlinear Systems. 2nd ed. Upper Saddle River, NJ: Prentice Hall, 1996.

[19] J. K. Hale and S. M. V. Lunel, Introduction to Functional Differential Equations, ser. Applied Mathematical Sciences. New York, NY: Springer Verlag, 1993, vol. 99.

[20] T. A. Burton, Stability and Periodic Solutions of Ordinary and functional differential equations, ser. Mathematics in Science and Engineering. Orlando, Florida: Academic Press, 1985, vol. 178.

[21] UCB, LBNL, and VINT, "Network simulator ns (version 2)," http://www.isi.edu/nsnam/ns/.

[22] A. S. Tanenbaum, Computer Networks. 3rd ed. Upper Saddle River, NJ: Prentice Hall, 1996.

[23] S. Floyd, M. Handley, J. Padhye, and J. Widmer, "Equation-based congestion control for unicast applications," in Proc. of ACM SIGCOMM Conf., August 2000, pp. 45-58. 